\title{
Direct writing of room temperature and zero field skyrmion lattices by a scanning local magnetic field
}

Senfu Zhang, Junwei Zhang, Qiang Zhang, Craig Barton, Volker Neu, Yuelei Zhao, Zhipeng Hou, Yan Wen, Chen Gong, Olga Kazakova, Wenhong Wang, Yong Peng, Dmitry A. Garanin, Eugene M. Chudnovsky, and Xixiang Zhang

Citation: Appl. Phys. Lett. 112, 132405 (2018); doi: 10.1063/1.5021172

View online: https://doi.org/10.1063/1.5021172

View Table of Contents: http://aip.scitation.org/toc/apl/112/13

Published by the American Institute of Physics

\section{Articles you may be interested in}

Observation of large exchange bias and topological Hall effect in manganese nitride films Applied Physics Letters 112, 132402 (2018); 10.1063/1.5025147

CMOS compatible $\mathrm{W} / \mathrm{CoFeB} / \mathrm{MgO}$ spin Hall nano-oscillators with wide frequency tunability Applied Physics Letters 112, 132404 (2018); 10.1063/1.5022049

Realization of zero-field skyrmions with high-density via electromagnetic manipulation in $\mathrm{Pt} / \mathrm{Co} / \mathrm{Ta}$ multilayers Applied Physics Letters 111, 202403 (2017); 10.1063/1.5001322

Skyrmion dynamics in width-varying nanotracks and implications for skyrmionic applications Applied Physics Letters 111, 202406 (2017); 10.1063/1.5005953

Spin-orbit torque-induced switching in ferrimagnetic alloys: Experiments and modeling Applied Physics Letters 112, 062401 (2018); 10.1063/1.5017738

Evolution of the spin hall magnetoresistance in $\mathrm{Cr}_{2} \mathrm{O}_{3} / \mathrm{Pt}$ bilayers close to the Néel temperature Applied Physics Letters 112, 132401 (2018); 10.1063/1.5019934

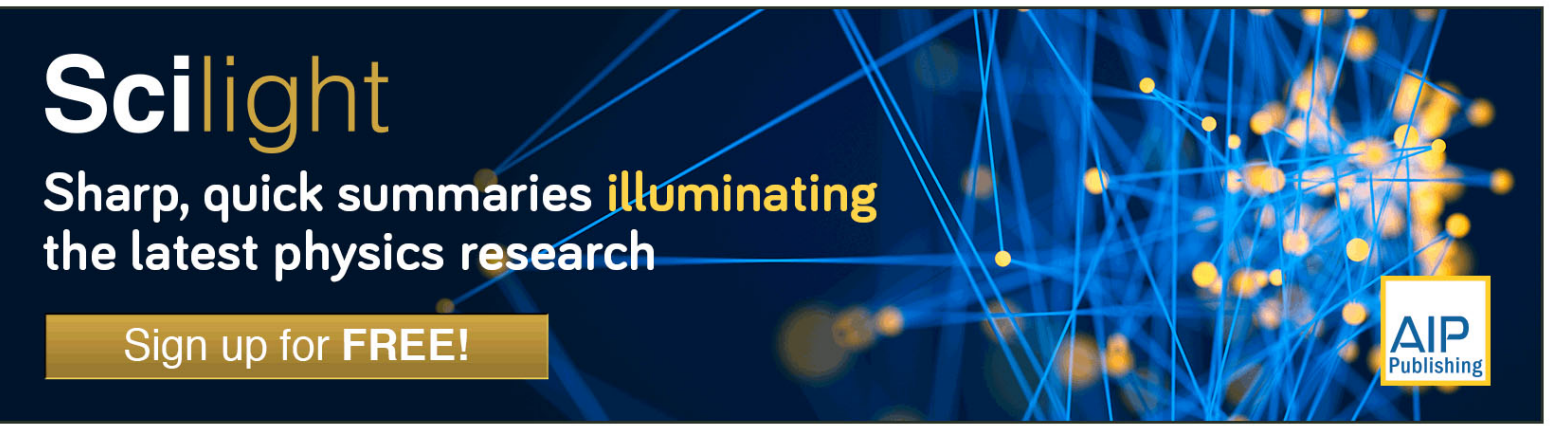




\title{
Direct writing of room temperature and zero field skyrmion lattices by a scanning local magnetic field
}

\author{
Senfu Zhang, ${ }^{1}$ Junwei Zhang, ${ }^{1,2}$ Qiang Zhang, ${ }^{1}$ Craig Barton, ${ }^{3}$ Volker Neu, ${ }^{4}$ Yuelei Zhao, ${ }^{1}$ \\ Zhipeng Hou, ${ }^{1,5}$ Yan Wen, ${ }^{1}$ Chen Gong, ${ }^{1}$ Olga Kazakova, ${ }^{3}$ Wenhong Wang, ${ }^{5}$ Yong Peng, ${ }^{2}$ \\ Dmitry A. Garanin, ${ }^{6}$ Eugene M. Chudnovsky, ${ }^{6}$ and Xixiang Zhang ${ }^{1, a)}$ \\ ${ }^{1}$ Physical Science and Engineering Division (PSE), King Abdullah University of Science and Technology \\ (KAUST), Thuwal 23955-6900, Saudi Arabia \\ ${ }^{2}$ Key Laboratory for Magnetism and Magnetic Materials of Ministry of Education, Lanzhou University, \\ Lanzhou 730000, People's Republic of China \\ ${ }^{3}$ National Physical Laboratory, Hampton Road, Teddington TW11 OLW, United Kingdom \\ ${ }^{4}$ IFW Dresden, Institute for Metallic Materials, Helmholtzstrasse 20, 01069 Dresden, Germany \\ ${ }^{5}$ National Laboratory for Condensed Matter Physics, Institute of Physics, Chinese Academy of Sciences, \\ Beijing 100190, China \\ ${ }^{6}$ Physics Department, Lehman College and Graduate School, The City University of New York, \\ 250 Bedford Park Boulevard West, Bronx, New York 10468-1589, USA
}

(Received 2 January 2018; accepted 24 February 2018; published online 29 March 2018)

\begin{abstract}
Magnetic skyrmions are topologically protected nanoscale spin textures exhibiting fascinating physical behaviors. Recent observations of room temperature skyrmions in sputtered multilayer films are an important step towards their use in ultra-low power devices. Such practical applications prefer skyrmions to be stable at zero magnetic fields and room temperature. Here, we report the creation of skyrmion lattices in $\mathrm{Pt} / \mathrm{Co} / \mathrm{Ta}$ multilayers by a scanning local field using magnetic force microscopy tips. We also show that those newly created skyrmion lattices are stable at both room temperature and zero fields. Lorentz transmission electron microscopy measurements reveal that the skyrmions in our films are of Néel-type. To gain a deeper understanding of the mechanism behind the creation of a skyrmion lattice by the scanning of local fields, we perform micromagnetic simulations and find the experimental results to be in agreement with our simulation data. This study opens another avenue for the creation of skyrmion lattices in thin films. Published by AIP Publishing. https://doi.org/10.1063/1.5021172
\end{abstract}

A magnetic skyrmion is a particle-like topologically protected structure and is one of the smallest magnetic structures that can be displaced by a very low current density. ${ }^{1-8}$ Thus, skyrmions hold great promise for the next generation low power nano-electronic devices, such as non-volatile storage, ${ }^{3,4,6,7,9,10}$ logic devices, ${ }^{11}$ and spin transfer nano-oscillators. ${ }^{12,13}$

Skyrmions were originally observed in B20 materials ${ }^{14-24}$ that have a non-centrosymmetric crystalline structure. In those materials, Dzyaloshinskii-Moriya interaction (DMI) originating from the structure plays a critical role in the formation of Bloch-type skyrmions. However, skyrmions in these materials exist only at low temperature and in the presence of an external magnetic field. ${ }^{14-24}$ Fortunately, DMI can also be produced by the breaking of inversion symmetry at the interfaces of ferromagnetic layers and heavy metal layers with large spin-orbit coupling, and this interfacial DMI can also be employed for the creation of skyrmions although these skyrmions are Néel-type rather than Blochtype. ${ }^{8,25}$ Such Néel-type skyrmions were first identified in epitaxial Fe or FePd monolayers on heavy metal $\operatorname{Ir}(111)$ substrates at very low temperature and high magnetic fields. ${ }^{26-28}$ To create room temperature skyrmions suitable for industrial applications, magnetic thin films with perpendicular magnetic anisotropy (PMA), in combination with heavy metals, such as $\mathrm{Ta}^{10,29-31}$ and $\mathrm{Pt}^{32-41}$ have been fabricated using sputtering. Skyrmions with sizes ranging from microns to

${ }^{a)}$ E-mail: xixiang.zhang@kaust.edu.sa $\sim 100 \mathrm{~nm}$ were observed in these thin-film stacks at room temperature. Thus, most recent studies have focused on this class of materials. Moreover, current induced motion of the skyrmions has also been observed in $\mathrm{Pt} / \mathrm{Co} / \mathrm{Ta}$ and similar systems. ${ }^{39,40}$ In the $\mathrm{Pt} / \mathrm{Co} / \mathrm{Ta}$ system, $\mathrm{Pt}$ and Ta have large, opposite spin Hall angles, making it possible to inject a large spin current into the ferromagnetic Co layer to drive the motion of skyrmions. Since large spin currents can result in faster skyrmion motions, the $\mathrm{Pt} / \mathrm{Co} / \mathrm{Ta}$ system might be one of the most suitable candidates for industrial applications.

For both fundamental investigations and practical applications, the first and most critical task is to identify reliable and simple technique(s) to create skyrmions. Moreover, skyrmions that are stable at room temperature and zero-magnetic fields are of additional advantage. ${ }^{8,25}$ To date, a number of techniques have been developed to generate skyrmions effectively, but it has been found that either the out-of-plane field or the geometric confining potential is required to stabilize the skyrmions. ${ }^{5,10,29,32,34,40}$ Magnetic force microscopy (MFM) ${ }^{23,37,40}$ is one of the typical methods to observe the skyrmion textures at the sample surface with a high spatial resolution. Here, we showed that MFM was not only an "eye" to observe the skyrmions but also a "hand" to create them in $[\operatorname{Pt}(4 \mathrm{~nm}) /$ $\mathrm{Co}(1.3 \mathrm{~nm}) / \mathrm{Ta}(1.9 \mathrm{~nm})]_{\mathrm{t}}$ multilayer stacks.

First, the multilayer stacks $\mathrm{Ta}(4.7 \mathrm{~nm}) /[\mathrm{Pt}(4 \mathrm{~nm}) / \mathrm{Co}$ $(1.3 \mathrm{~nm}) / \mathrm{Ta}(1.9 \mathrm{~nm})]_{\mathrm{t}}$ were deposited by DC magnetron sputtering at room temperature. Then, High resolution high angle annular dark field scanning transmission electron microscopy 
(HADDF-STEM) was performed to examine the crosssection of a $t=10$ multilayer as shown in Fig. 1(a). The inset shows an enlarged view of the cross-section in which the Co layer is represented by a dark contrast. Energy dispersive $\mathrm{X}$-ray (EDX) microanalysis shows clear oscillations in line scans of $\mathrm{Co} K \alpha$, Pt $\mathrm{K} \alpha$, and Ta $\mathrm{K} \alpha$ along the growth direction of the film (Fig. S1). The magnetic properties of the multilayers were measured using SQUID-VSM at room temperature. Figure 1(b) shows the Out-of-plane hysteresis loops (see the in-plane loops in Fig. S2) for three samples with layer repetition numbers, $t=5,10$, and 20 , which indicate that all our samples are PMA and the saturation field increases with the increase in the layer repetition number.

To observe domain structures of the samples, MFM experiments were performed. The principle of MFM is that when a magnetic sample surface is scanned by a sharp magnetized tip, the magnetic interactions between the tip moments and the stray field of the surface can be detected and recorded. Thus, there is an unavoidable distortion of the sample's magnetic structure due to the stray field of the magnetized tip, especially for the soft magnetic materials or nanoparticles. ${ }^{42,43}$ To image an "intrinsic" domain structure of a sample, we tried to alleviate the perturbations by choosing tips with a lower moment and a larger tip-sample distance. Here, we will demonstrate that this "adverse effect" can be used to directly write skyrmions by choosing proper tips and tuning the tip-sample distance using the tapping/lift mode. Figure 1(c) shows the $z$ component of the stray magnetic fields as a function of the distance to the tip apex, which was obtained by using the tip transfer function method (see the details in supplementary material SI1), ${ }^{44-48}$ for a low moment MFM tip1 (Bruker MESP-LM-V2) and a large moment tip2 (Nanosensor PPP-MFMR). It can be seen that the stray fields could reach about 570 Oe and 1350 Oe at the apex of tip1 and tip 2, respectively. However, the stray field decays quickly with the distance and becomes very weak when the distance is larger than $50 \mathrm{~nm}$. In the tapping/lift mode, the topography and magnetic contrasts are separated by two scans. The topography of the sample is first obtained using the tapping mode, in which the MFM tip is in contact with the film surface and a much stronger local magnetic field is applied to the sample. Thus, the first scan in the tapping mode could be used to incise the labyrinth domain into skyrmions. A magnetic image is subsequently obtained using the lift mode at a pre-set lift height of larger than $50 \mathrm{~nm}$. Micromagnetic simulations (supplementary material SI3) showed that to write the skyrmions, the local magnetic field should be slightly larger than the saturation field of the sample. Fortunately, we were able to control the saturation fields of our samples by tuning the thickness of individual layers and the repetition number.

First, tip1 that has a weaker stray field was used to scan the samples with a lift height of $100 \mathrm{~nm}$. Figures $1(\mathrm{~d})-1$ (f) show MFM images for samples with $t=5,10$, and 20 in the remnant state, after removal of the saturation field applied perpendicularly to the film plane. These images show that the skyrmion lattice phase appeared in the $t=5$ sample with a skyrmion size of about $200 \mathrm{~nm}$, while the labyrinth domain structures were observed for samples with $t=10$ and 20. The labyrinth domains observed for samples with $t=10$ were similar to those observed in the Lorentz transmission electron microscopy (L-TEM) images [Fig. S8(c)], revealing that the stray field of tip1 was not strong enough to induce a visible distortion of the magnetic structure during the scan in the tapping mode or that the labyrinth domain structures were restored to minimize the total magnetic energy after the MFM tip moved away. The distortion for the $t=20$ sample should be negligible for the even larger saturation field of $\sim 1200$ Oe [Fig. 1(b)]. However, for the $t=5$ sample whose saturation field was relatively small [ 440 Oe, Fig. 1(b)], the stray field became strong enough to completely change the domain structure. In this case, skyrmion lattices were created. Therefore, the stray field at the apex of tip1 might be between 440 Oe and $660 \mathrm{Oe}$, which agree well with the value of about 570 Oe derived from the tip calibration procedure in Fig. 1(c). To create skyrmion lattices in samples those have larger saturation fields, tip2, whose stray field at the tip apex is slightly larger than the saturation field of the $t=20$ sample, was used to scan the three samples again. Figure $1(\mathrm{~g})$ shows the result for the $t=20$ sample, in which the skyrmion
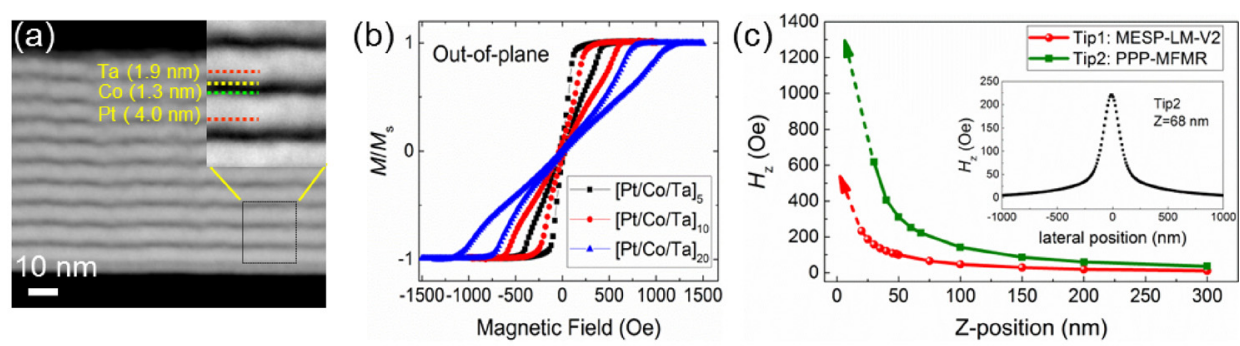

Tip1
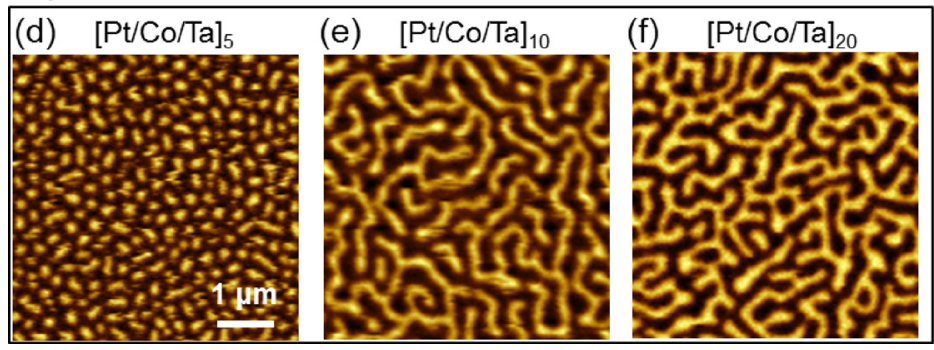

Tip2

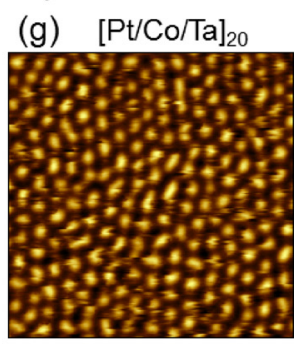

FIG. 1. (a) HADDF-STEM image of a cross-section of a $\mathrm{Ta}(4.7 \mathrm{~nm}) /$ $[\mathrm{Pt}(4 \mathrm{~nm}) / \mathrm{Co}(1.3 \mathrm{~nm}) / \mathrm{Ta}(1.9 \mathrm{~nm})]_{10}$ multilayer sample. The inset shows an enlarged view, and the $\mathrm{Ta} / \mathrm{Co}, \mathrm{Co} / \mathrm{Pt}$, and $\mathrm{Pt} / \mathrm{Ta}$ interfaces are marked by yellow, green, and red dashed lines. (b) Perpendicular hysteresis loops for samples with layer repetition numbers of $t=5,10$, and 20. (c) Z component of the stray field as a function of the distance to the tip apex for the two MFM tips. The inset shows the $z$ component of the stray field vs lateral distance at $\mathrm{z}=68 \mathrm{~nm}$ for tip2. (d)-(f) MFM images for the three samples, obtained at a lift height of $100 \mathrm{~nm}$ by tip1 (Bruker MESP-LM-V2). (g) MFM image for the $t=20$ sample scanned by Tip2 (Nanosensor PPPMFMR). 

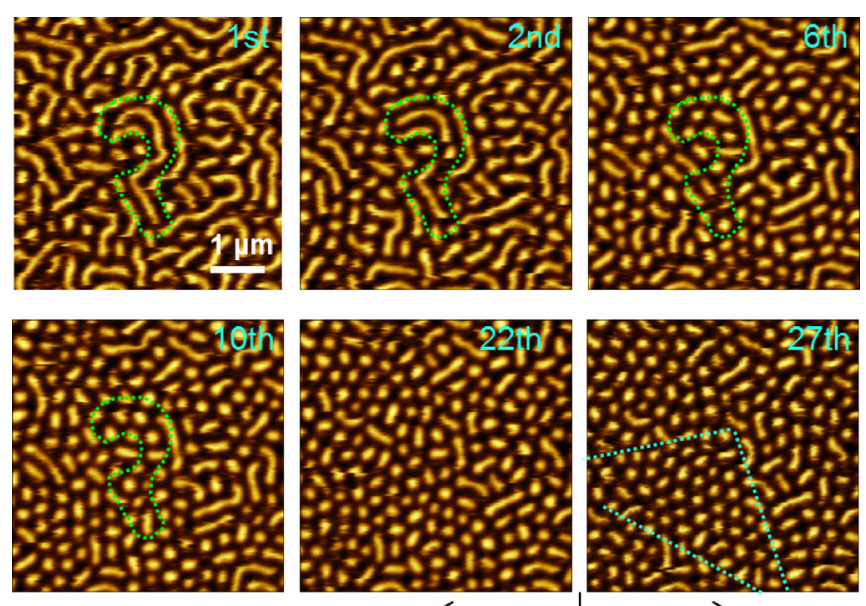

Lift height: $100 \mathrm{~nm}$

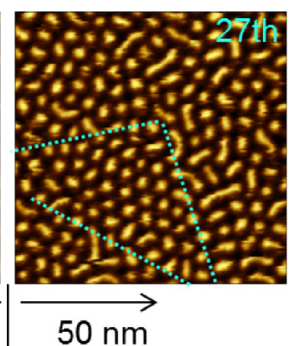

FIG. 2. 6 representative MFM images taken during scans (in total, 27 scans were performed) over the same area of a $t=20$ sample with tip2 (Nanosensor PPP-MFMR). The lift heights were $50 \mathrm{~nm}$ for the 27th scan and $100 \mathrm{~nm}$ for others. Multimedia view: https://doi.org/10.1063/1.5021172.1

lattice is clearly seen, as expected. Such a strong stray field certainly influenced the magnetic domain structure of the $t=10$ sample significantly [Fig. S9(a)]. As expected, the resultant changes in the domain structures of the $t=5$ film were then more significant [Fig. S9(b)].

We have created skyrmion lattices at room temperature and under zero external magnetic fields without any geometric confining potential. More importantly, the stable skyrmion lattices could be created just by a single scan of the MFM tip in the tapping mode. However, to better understand the creation process, it would be preferable if the skyrmions could be created gradually. Since tips may slightly differ, even from the same batch, we therefore scanned the $t=20$ sample with more tips from the same batch (tip2: Nanosensor PPP-MFMR). Eventually, we found one tip that was relatively weak magnetically and could create skyrmions gradually by repeating the scanning process over the same area as shown in Fig. 2 (Multimedia view). It clearly shows that after the first scan, a mixed state of skyrmions and worm domains appeared. By repeating the scans over the same area, more and more skyrmions were created and the lengths of worm-like domains were shortened. The area enclosed with the green dotted lines shows a typical process of how the worm domain was cut into individual skyrmions. More interestingly, as more and more skyrmions were created, a symmetric hexagonal skyrmion lattice was formed gradually. To obtain a clearer MFM image, the lift height was decreased to $50 \mathrm{~nm}$ at the $27 \mathrm{th}$ scan. It is evident that an almost perfect hexagonal skyrmion lattice was formed in the area enclosed by the triangle.

From the above results, we showed that in order to create the skyrmion lattices, the relative strength of the stray field of a MFM tip to the saturation field of the film should be within a certain range. In the following, by applying an external field, we further demonstrate that skyrmions could also be created by a tip whose stray field is smaller than the saturation field of the sample. As shown in Fig. 1(e), the stray field from tip1 was too weak to create skyrmions on the $t=10$ sample. We scanned the samples many times and found that the labyrinth domain structure remained the same as shown in Fig. 3(a). Although a few spontaneous isolated skyrmions were observed, as indicated by the green dashed circles, the labyrinth domain structure was predominant. We then applied a 110 Oe out-of-plane field along the $-z$ direction, the same direction of the tip's stray field. The overall domain structures almost remained unchanged although three more skyrmions (indicated by white dashed circles) were created at the edges of the labyrinth domain as shown in Fig. 3(b). As the magnetic field increased to $165 \mathrm{Oe}$, the labyrinth domain began to break into discrete skyrmions and a mixed state of skyrmions, and snake-like domains appeared as shown in Fig. 3(c). To further assess the stability of the skyrmions and also prove that these skyrmions are created by the MFM tip with the assistance of the external field, we set the applied field to zero and scanned the sample again as shown in Fig. 3(d). The scanned area in Fig. 3(d) covered the area enclosed in the square in Fig. 3(c), as well as an adjacent area that was never scanned by the tip before. It is clearly seen that the magnetic structures in the two squares are nearly the same, except the change in the size because of the different field strength, indicating that the created skyrmions were also stable at zero magnetic fields. The labyrinth domain structure outside the square in Fig. 3(d) is also very similar to that shown in Figs. 3(a) and 3(b), suggesting that without the scanning process using the MFM tip, the external field with a maximum of $165 \mathrm{Oe}$ in our experiment did not create skyrmions.

L-TEM measurements (see supplementary material SI3) proved that the skyrmions in our samples are of Néel type. To gain a deeper understanding of the skyrmions' creation mechanism, micromagnetic simulations were performed using mumax $^{3}$ software $^{49}$ (see the details in supplementary material SI2). Figure 4(a) shows the initial relaxed state of a $2 \times 2 \mu \mathrm{m}^{2}$ square system. To simplify the simulations, a
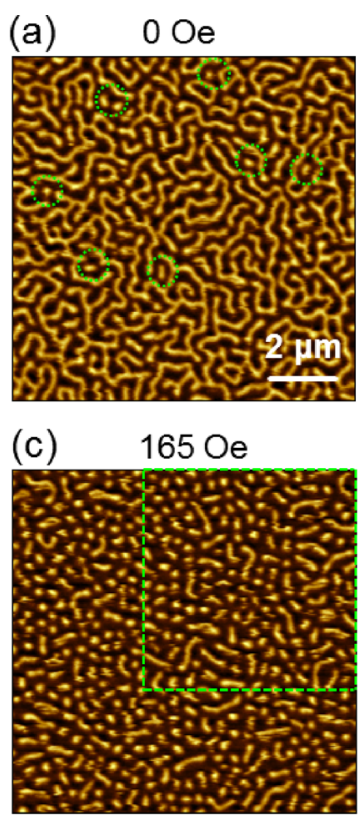
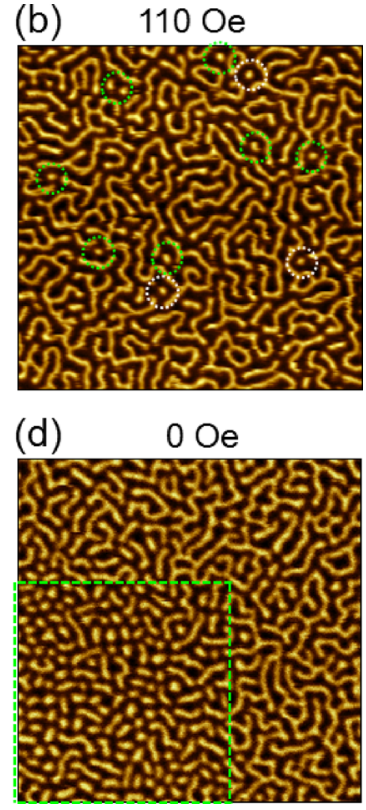

FIG. 3. MFM images with an external field of (a) 0 Oe, (b) 110 Oe, and (c) 165 Oe on the $t=10$ sample using tip1 (Bruker MESP-LM-V2). The green dashed circles indicate some spontaneous isolated skyrmions. The white dashed circles indicate the newly appeared skyrmions. (d) MFM images after the removal of the external field. The area enclosed in the green square is the same area as in the square of (c). 
1 st scan
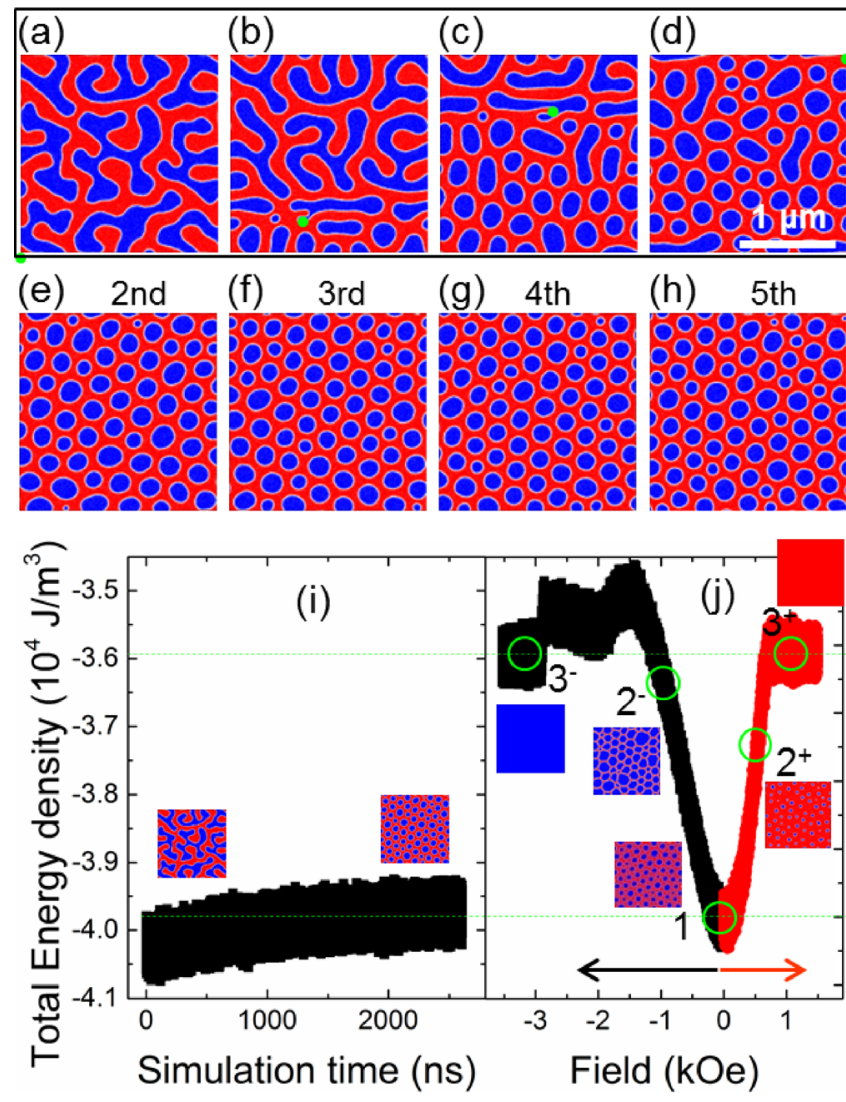

FIG. 4. (a) Initial relaxed state of the system. The blue/red contrast indicates magnetization along the negative/positive $z$ direction. (a)-(d) Evolution of the magnetic domain structures during the scanning of the local magnetic field. (e)-(h) The magnetic structures for the following 4 time scan with the same process. (i) Total energy of the system as a function of simulation time, starting from the labyrinth domains in (a) to a stable skyrmion lattice state after the fifth scan in (h). (j) Total energy of the system as a function of the perpendicular magnetic field with initial states of the skyrmion lattice. The red/black arrows indicate the magnetic field decrease/increase from 0 Oe. The insets show the magnetic structure of the film under different applied magnetic fields. Multimedia view: https://doi.org/10.1063/1.5021172.2

uniform magnetic field confined within a circle of $50 \mathrm{~nm}$ in diameter along the $+\mathrm{z}$ direction was applied to represent the local magnetic field of a MFM tip. We then performed a scanning of the local field from the lower left corner to the upper right corner, with a step size of $40 \mathrm{~nm}$ in both $\mathrm{x}$ and $\mathrm{y}$ directions. Figures 4(a)-4(d) (Multimedia view) show the evolution of the magnetic domain structures during the scanning process, in which the positions of the local magnetic field are highlighted by solid green circles. It is apparent that the local magnetic field "cut" the labyrinth domains [Fig. 4(a)] into small objects, specifically skyrmions. It is worth noting that after the first scan, there were still some stripe domains in the film. To reach a phase solely composed of skyrmions, we performed 4 more scans continuously over the same area as shown in Figs. 4(e)-4(h). One can clearly see that the worm-like domains were cut into a few skyrmions, creating a hexagonal skyrmion lattice state.

To understand the evolution and stability of the domain structure under the scanning of the local field, we calculated the total energy of the sample, by using $E_{T}=E_{\text {anis }}+E_{\text {demag }}+E_{e x}$ $+E_{D M I}$, where $E_{\text {anis }}, E_{\text {demag }}, E_{e x}$, and $E_{D M I}$ are the anisotropy, demagnetization, exchange, and DMI energy. It is important to note that to study the intrinsic properties and compare the energy evolution under the same conditions, the Zeeman energy (which strongly depends on the external field) is not considered in this case. Figure 4(i) shows the variation of the system total energy with the magnetic structures starting from a labyrinth-shaped [Fig. 4(a)] to a stable skyrmion lattice after the fifth scan [Fig. 4(h)]. Figure 4(i) clearly shows that the total energy for a skyrmion lattice configuration is only slightly higher than that of a spontaneous labyrinth domain state. To further investigate the stability of the skyrmion lattice in the magnetic field, out-ofplane external fields are applied to the lattice system. Figure 4(j) shows the relationship between total energy and the positive/ negative magnetic fields. One can see that the total energy first increases with the increase in field strength and then decreases to a constant value that corresponds to a state of magnetic saturation. The insets of Fig. 4(j) show magnetic structures of the film under different applied magnetic fields. We noticed that when the applied magnetic field was in the positive direction, i.e., antiparallel to the magnetization of the core of skyrmions, the size of the skyrmions shrank $\left(2^{+}\right)$with an increasing field strength. When the field was strong enough, an annihilation of the skyrmions occurred, and finally, the sample saturated into a ferromagnetic phase with the magnetization aligning with the field direction $\left(3^{+}\right)$. In contrast, when the field was applied in the negative direction, i.e., parallel to the core magnetization, the size of the skyrmions increased $\left(2^{-}\right)$, with the edges of the skyrmions squeezing together. When the field was strong enough, the boundary between the skyrmions was destroyed, and eventually, the sample was saturated into a ferromagnetic phase $\left(3^{-}\right)$. In short, the skyrmion lattice state is a metastable state.

In summary, we were able to create skyrmion lattices in $[\mathrm{Pt} / \mathrm{Co} / \mathrm{Ta}]_{\mathrm{t}}$ multilayer stacks with PMA by scanning the film with MFM tips, using the stray field of the MFM tip as a "knife" to "cut" the labyrinth domain into skyrmions in the tapping mode. The skyrmion lattices that we created were found to be stable in zero magnetic fields and at room temperature. We further demonstrated that an external magnetic field could facilitate the creation of skyrmions. In situ L-TEM measurements showed that the skyrmions present in those films were of Néel-type. The creation process of the skyrmion lattice by the scanning of the MFM tips was reproduced with micromagnetic simulation. In addition, the simulation indicates that the skyrmion lattice state is a metastable state.

See supplementary material for the details of the stray field measurement, additional simulations, and L-TEM imaging.

This publication is based on the research supported by the King Abdullah University of Science and Technology (KAUST), Office of Sponsored Research (OSR) under the Award No. OSR-2016-CRG5-2977. This work was also supported in part by the European Metrology Research Programme (EMRP) and EMRP participating countries under the EMPIR Project No. 15SIB06-Nanomag: Nanoscale traceable magnetic field measurements.

\footnotetext{
${ }^{1}$ I. Dzyaloshinsky, J. Phys. Chem. Solids 4, 241 (1958).

${ }^{2}$ T. Moriya, Phys. Rev. 120, 91 (1960).
} 
${ }^{3}$ A. Fert, V. Cros, and J. Sampaio, Nat. Nanotechnol. 8, 152 (2013).

${ }^{4}$ J. Iwasaki, M. Mochizuki, and N. Nagaosa, Nat. Nanotechnol. 8, 742 (2013).

${ }^{5}$ N. Nagaosa and Y. Tokura, Nat. Nanotechnol. 8, 899 (2013).

${ }^{6}$ J. Sampaio, V. Cros, S. Rohart, A. Thiaville, and A. Fert, Nat. Nanotechnol. 8, 839 (2013).

${ }^{7}$ X. Yu, N. Kanazawa, W. Zhang, T. Nagai, T. Hara, K. Kimoto, Y. Matsui, Y. Onose, and Y. Tokura, Nat. Commun. 3, 988 (2012).

${ }^{8}$ W. Jiang, G. Chen, K. Liu, J. Zang, S. G. te Velthuis, and A. Hoffmann, Phys. Rep. 704, 1 (2017).

${ }^{9}$ R. Tomasello, E. Martinez, R. Zivieri, L. Torres, M. Carpentieri, and G. Finocchio, Sci. Rep. 4, 6784 (2014).

${ }^{10}$ G. Yu, P. Upadhyaya, Q. Shao, H. Wu, G. Yin, X. Li, C. He, W. Jiang, X. Han, P. K. Amiri, and K. Wang, Nano Lett. 17, 261 (2016).

${ }^{11}$ X. Zhang, M. Ezawa, and Y. Zhou, Sci. Rep. 5, 9400 (2015).

${ }^{12}$ S. Zhang, J. Wang, Q. Zheng, Q. Zhu, X. Liu, S. Chen, C. Jin, Q. Liu, C. Jia, and D. Xue, New J. Phys. 17, 023061 (2015).

${ }^{13}$ F. Garcia-Sanchez, J. Sampaio, N. Reyren, V. Cros, and J. Kim, New J. Phys. 18, 075011 (2016).

${ }^{14}$ S. Grigoriev, V. Dyadkin, E. Moskvin, D. Lamago, T. Wolf, H. Eckerlebe, and S. Maleyev, Phys. Rev. B 79, 144417 (2009).

${ }^{15}$ S. Mühlbauer, B. Binz, F. Jonietz, C. Pfleiderer, A. Rosch, A. Neubauer, R. Georgii, and P. Böni, Science 323, 915 (2009).

${ }^{16}$ C. Pappas, E. Lelievre-Berna, P. Falus, P. Bentley, E. Moskvin, S. Grigoriev, P. Fouquet, and B. Farago, Phys. Rev. Lett. 102, 197202 (2009).

${ }^{17}$ F. Jonietz, S. Mühlbauer, C. Pfleiderer, A. Neubauer, W. Münzer, A. Bauer, T. Adams, R. Georgii, P. Böni, and R. Duine, Science 330, 1648 (2010).

${ }^{18}$ W. Münzer, A. Neubauer, T. Adams, S. Mühlbauer, C. Franz, F. Jonietz, R. Georgii, P. Böni, B. Pedersen, and M. Schmidt, Phys. Rev. B 81, 041203 (2010).

${ }^{19}$ X. Yu, Y. Onose, N. Kanazawa, J. Park, J. Han, Y. Matsui, N. Nagaosa, and Y. Tokura, Nature 465, 901 (2010).

${ }^{20}$ N. Kanazawa, Y. Onose, T. Arima, D. Okuyama, K. Ohoyama, S. Wakimoto, K. Kakurai, S. Ishiwata, and Y. Tokura, Phys. Rev. Lett. 106, 156603 (2011).

${ }^{21}$ X. Yu, N. Kanazawa, Y. Onose, K. Kimoto, W. Zhang, S. Ishiwata, Y. Matsui, and Y. Tokura, Nat. Mater. 10, 106 (2011).

${ }^{22}$ A. Tonomura, X. Yu, K. Yanagisawa, T. Matsuda, Y. Onose, N. Kanazawa, H. S. Park, and Y. Tokura, Nano Lett. 12, 1673 (2012).

${ }^{23}$ P. Milde, D. Köhler, J. Seidel, L. Eng, A. Bauer, A. Chacon, J. Kindervater, S. Mühlbauer, C. Pfleiderer, and S. Buhrandt, Science 340, 1076 (2013).

${ }^{24}$ K. Shibata, X. Yu, T. Hara, D. Morikawa, N. Kanazawa, K. Kimoto, S. Ishiwata, Y. Matsui, and Y. Tokura, Nat. Nanotechnol. 8, 723 (2013).

${ }^{25}$ A. Fert, N. Reyren, and V. Cros, Nat. Rev. Mater. 2, 17031 (2017).

${ }^{26}$ S. Heinze, K. von Bergmann, M. Menzel, J. Brede, A. Kubetzka, R. Wiesendanger, G. Bihlmayer, and S. Blügel, Nat. Phys. 7, 713 (2011).

${ }^{27}$ N. Romming, C. Hanneken, M. Menzel, J. E. Bickel, B. Wolter, K. von Bergmann, A. Kubetzka, and R. Wiesendanger, Science 341, 636 (2013).
${ }^{28}$ A. Schlenhoff, P. Lindner, J. Friedlein, S. Krause, R. Wiesendanger, M. Weinl, M. Schreck, and M. Albrecht, ACS Nano 9, 5908 (2015).

${ }^{29}$ W. Jiang, P. Upadhyaya, W. Zhang, G. Yu, M. B. Jungfleisch, F. Y. Fradin, J. E. Pearson, Y. Tserkovnyak, K. L. Wang, and O. Heinonen, Science 349, 283 (2015).

${ }^{30}$ G. Yu, P. Upadhyaya, X. Li, W. Li, S. K. Kim, Y. Fan, K. L. Wong, Y. Tserkovnyak, P. K. Amiri, and K. L. Wang, Nano Lett. 16, 1981 (2016).

${ }^{31}$ W. Jiang, X. Zhang, G. Yu, W. Zhang, X. Wang, M. B. Jungfleisch, J. E. Pearson, X. Cheng, O. Heinonen, and K. L. Wang, Nat. Phys. 13, 162 (2017).

${ }^{32}$ G. Chen, A. Mascaraque, A. T. N'Diaye, and A. K. Schmid, Appl. Phys. Lett. 106, 242404 (2015).

${ }^{33}$ H. Yang, A. Thiaville, S. Rohart, A. Fert, and M. Chshiev, Phys. Rev. Lett. 115, 267210 (2015).

${ }^{34}$ O. Boulle, J. Vogel, H. Yang, S. Pizzini, D. de Souza Chaves, A. Locatelli, T. O. Menteş, A. Sala, L. D. Buda-Prejbeanu, and O. Klein, Nat. Nanotechnol. 11, 449 (2016).

${ }^{35}$ C. Moreau-Luchaire, C. Moutafis, N. Reyren, J. Sampaio, C. Vaz, N. Van Horne, K. Bouzehouane, K. Garcia, C. Deranlot, and P. Warnicke, Nat. Nanotechnol. 11, 444 (2016).

${ }^{36}$ J. F. Pulecio, A. Hrabec, K. Zeissler, R. M. White, Y. Zhu, and C. H. Marrows, preprint arXiv:1611.06869 (2016).

${ }^{37}$ A. Soumyanarayanan, M. Raju, A. L. Gonzalez Oyarce, A. K. C. Tan, M.-Y. Im, A. P. Petrovic, P. Ho, K. H. Khoo, M. Tran, C. K. Gan, F. Ernult, and C. Panagopoulos, Nat. Mater. 16, 898 (2017).

${ }^{38}$ R. Wiesendanger, Nat. Rev. Mater. 1, 16044 (2016).

${ }^{39}$ S. Woo, K. Litzius, B. Krüger, M.-Y. Im, L. Caretta, K. Richter, M. Mann, A. Krone, R. M. Reeve, and M. Weigand, Nat. Mater. 15, 501 (2016).

${ }^{40}$ W. Legrand, D. Maccariello, N. Reyren, K. Garcia, C. Moutafis, C. Moreau-Luchaire, S. Collin, K. Bouzehouane, V. Cros, and A. Fert, Nano Lett. 17, 2703 (2017).

${ }^{41}$ S. D. Pollard, J. A. Garlow, J. Yu, Z. Wang, Y. Zhu, and H. Yang, Nat. Commun. 8, 14761 (2017).

${ }^{42}$ M. Kleiber, F. Kümmerlen, M. Löhndorf, A. Wadas, D. Weiss, and R. Wiesendanger, Phys. Rev. B 58, 5563 (1998).

${ }^{43}$ M. Jaafar, A. Asenjo, and M. Vazquez, IEEE Trans. Nanotechnol. 7(3), 245-250 (2008).

${ }^{44}$ P. van Schendel, H. Hug, B. Stiefel, S. Martin, and H.-J. Güntherodt, J. Appl. Phys. 88, 435 (2000).

${ }^{45}$ S. Vock, C. Hengst, M. Wolf, K. Tschulik, M. Uhlemann, Z. Sasvári, D. Makarov, O. Schmidt, L. Schultz, and V. Neu, Appl. Phys. Lett. 105(17), 172409 (2014).

${ }^{46}$ C. F. Reiche, S. Vock, V. Neu, L. Schultz, B. Büchner, and T. Mühl, New J. Phys. 17(1), 013014 (2015).

${ }^{47}$ V. Panchal, H. Corte-León, B. Gribkov, L. A. Rodriguez, E. Snoeck, A. Manzin, E. Simonetto, S. Vock, V. Neu, and O. Kazakova, Sci. Rep. 7(1), 7224 (2017).

${ }^{48}$ N. Zingsem, F. Ahrend, S. Vock, D. Gottlob, I. Krug, H. Doganay, D. Holzinger, V. Neu, and A. Ehresmann, J. Phys. D: Appl. Phys. 50(49), 495006 (2017).

${ }^{49}$ A. Vansteenkiste, J. Leliaert, M. Dvornik, M. Helsen, F. Garcia-Sanchez, and B. Van Waeyenberge, AIP Adv. 4, 107133 (2014). 